Full Paper

\title{
Auswirkungen der Nacherntebehandlung mit Schellack auf Äpfel der Sorten Red Delicious und Fuji
}

\author{
Effects of the post-storage treatement with natural shellac on Red Delicious and Fuji apple varieties
}

Effetti del trattamento post-conservazione con gommalacca naturale sulle mele Red Delicious e Fuji

Angelo Zanella ${ }^{1}$, Alessia Panarese ${ }^{1}$

${ }^{1}$ Versuchszentrum Laimburg

\section{ABSTRACT}

Coating fruits with shellac after storage improves fruit appearance. Moreover, it could preserve better their intrinsic quality and prevent physiological disorders such as superficial scald. During two years the influence of the coating with shellac and of the related treatment process was investigated on apple cv. Red Delicious and Fuji at the Research Centre Laimburg taking into account the difference in quality, before the coating, after storage either in CAULO, CA-ULO with SmartFresh or in dynamic CA by means of chlorophyll fluorescence (DCACF). On treated Red Delicious a trend for lower water-loss and scald and for higher firmness was observed after shelf-life. On treated Fuji we could demonstrate that the thermal shock involved in the coating process induced lenticel breakdown, and not the coating itself.

\section{KEYWORDS}

shellac, Red Delicious, Fuji, DCA-CF, 1-MCP, lenticel breakdown, superficial scald

\section{CITE ARTICLE AS}

Zanella Angelo, Panarese Alessia (2019). Effects of the post-storage treatement with natural shellac on Red Delicious and Fuji apple varieties. Laimburg Journal $1 / 2019$ https://www.doi.org/10.23796/L/ 2019.006

\section{CORRESPONDING AUTHOR}

Angelo Zanella

Laimburg 6, Pfatten, I-39040 Auer

(BZ), Italien

angelo.zanella@laimburg.it +390471969540 


\section{EINLEITUNG}

Seit einigen Jahren sind Nacherntebehandlungen mit Wachsprodukten, welche den Früchten ein besseres Aussehen verleihen und aufgrund ihrer erhöhten Glanz- und Deckfarbenintensität dem Konsumenten attraktiver erscheinen lassen, weltweit im Obst- und Gemüsesektor verbreitet. Zudem wird die Lagerfähigkeit, besonders in jenen Fällen, wo eine kontinuierliche Kühlkette nur beschränkt möglich ist, verlängert, weshalb solche Nacherntebehandlungen besonders auf dem nordafrikanischen und arabischen Markt aber auch auf der iberischen Halbinsel sehr beliebt sind.

Am Versuchszentrum Laimburg wurden im Rahmen eines zweijährigen Versuches die Auswirkungen einer Nacherntebehandlung mit einem in der Praxis bereits angewandten Wachs bei den Sorten Red Delicious und Fuji untersucht. Bei dem genannten Produkt handelt es sich um Schellack, ein natürliches in Ethylalkohol gelöstes Polymer, welches nach erfolgreicher Applikation eine Schutzschicht um die Frucht bildet und diese dadurch attraktiver erscheinen lässt. Zudem wird die Frucht durch die Behandlung mit dem genannten Produkt semipermeabel für Gase, was eventuell eine Verlangsamung der Reifeprozesse und/oder eine Reduzierung der Wassertranspiration zur Folge haben könnte.

Ziel der am Versuchszentrum Laimburg durchgeführten Versuchsreihe war die Untersuchung der Auswirkungen einer solchen Behandlung an der Sorte Red Delicious, wobei eventuelle Qualitätsunterschiede zwischen behandelten und nicht behandelten Früchten bei unterschiedlichen Lagerbedingungen erkannt werden sollten. Neben den inneren Qualitätsparametern wurde dabei auch die Anfälligkeit der Früchte gegenüber Fäulnis, inneren Schäden und physiologischen Störungen, mit besonderem Augenmerk auf die gewöhnliche Schalenbräune, untersucht.

Zusätzlich wurde der Einfluss einer Wachsbehandlung auf die Entwicklung von Aromen und eventuellen Störaromen, vor allem nach der Lagerung bei sehr niedrig eingestellten Sauerstoffwerten, wie der DCA-CF Lagerung, evaluiert.

Das Prinzip der DCA-CF Lagertechnologie basiert auf der kontinuierlichen Messung der Chlorophyllfluoreszenz einer repräsentativen Apfelstichprobe mittels speziell dafür entwickelter Sensoren, wodurch eine
Einstellung von extrem niedrigen Sauerstoffwerten ermöglicht wird. Dadurch kann das Auftreten der gewöhnlichen Schalenbräune vollständig verhindert werden und auch Seneszenzprozesse werden dadurch stark verlangsamt (Zanella et al. 2005) [1] .

Bei den Versuchen mit der Sorte Fuji hingegen lag das prioritäre Ziel in der Ursachenfindung für das Auftreten der LentizellenSchwäche ('lenticel breakdown', Abb. 1) und darin, einen möglichen Zusammenhang mit der Schellack-Behandlung zu untersuchen.

Symptome dieser Physiopathologie wurden bei gewachsten Früchten beobachtet. Diese zeichneten sich durch dunkelbraune Flecken rund um die Lentizellen ohne das Vorhandensein von Pathogenen aus. Die Flecken vergrößern sich im weiteren Krankheitsverlauf aufgrund des Auftretens von sekundärer Fäulnis (Abb. 2). Die Lentizellen-Schwäche tritt hauptsächlich während der Nachreifung nach der Auslagerung auf.

Die angeführte Physiopathologie ist vor allem in letzter Zeit ins Visier der Forschung geraten, da sie seit ungefähr einem Jahrzehnt in bekannten Produktionsregionen wie Washington State (USA), besonders bei den Sorten Gala und Fuji (Kupferman, 2005) [2] erhebliche Schäden verursacht.

\section{MATERIALIEN UND METHODEN}

\section{UNTERSUCHUNGEN ZUR SORTE RED DELICIOUS}

Die Früchte der Sorte Red Delicious stammten aus einer Anlage des Versuchszentrums Laimburg, von Bäumen mit Spur-Typ Wachstum, Klon Red Chief, Pflanzjahr 1997. Die Äpfel wurden im ersten Jahr in zwei Pflücken geerntet, und zwar am 3. und 15. September 2009, und im Jahr 2010 an einem einzigen Termin, und zwar am 8. September. Zur Ernte wurden die chemisch-physikalischen Parameter Fruchtfleischfestigkeit, lösliche Trockensubstanz und titrierbare Säure von 45 Früchten mittels Pimprenelle (Setop, Frankreich) erhoben und der Stärkeabbau als Reife-Parameter durch das Einfärben der Stärke mit Lugol'scher Lösung visuell geschätzt (Laimburg Skala 1-5). Anschließend wurden die Früchte für sechs Monate bei $1.3^{\circ} \mathrm{C}$ unter verschiedenen Bedingungen gelagert: ULO (1\% O2 und 1.5\% CO2), DCA-CF (0.5\% $\mathrm{O} 2$ und 1.3\% CO2; siehe Zanella et al. (2005) [1] und ULO mit 1-MCP (SmartFresh, Agrofresh Inc.). Nach der Auslagerung wurden von jeder Variante (ULO, DCA-CF und
ULO+1-MCP) 90 gesunde Früchte einheitlicher Größe (70-95 mm) entnommen. 45 Früchte (15×3 Wiederholungen) jeder Variante wurden mit Schellack, Xedafos $L$ (Xeda International s.a., St. Andiol, Frankreich) mittels Tauchbad behandelt. Die restlichen Äpfel wurden nur mit Wasser behandelt und dienten als Kontrolle. Die Behandlung wurde wie folgt durchgeführt: Nach der Lagerungsperiode in den drei unterschiedlichen Lagerungsbedingungen wurden die noch kalten Früchte $\left(1.3^{\circ} \mathrm{C}\right)$ für 7 Minuten in $30^{\circ} \mathrm{C}$ warmes Wasser (laut Praxis) getaucht, danach mit Druckluft bei Raumtemperatur $\left(18^{\circ} \mathrm{C}\right)$ getrocknet und anschließend sofort mit Schellack behandelt. Für die Wachsbehandlung wurden die Früchte einschichtig in Kisten gelegt, kurz (ca. 1 Sek.) in die Wachslösung getaucht und anschließend getrocknet.

Nach 24 Stunden wurden die Äpfel einzeln gewogen und eine Woche lang unter Shelf Life Bedingungen bei $20^{\circ} \mathrm{C}$ und $60-70 \%$ RF (relative Luftfeuchte) gelagert. Nach der Shelf Life Periode wurden die Früchte für die Bestimmung des Gewichtsverlustes wiederum einzeln gewogen. Zudem wurden die äußeren Schäden visuell ausgewertet, die chemisch-physikalischen Parameter mittels Pimprenelle analysiert und die inneren Schäden beim Aufschneiden erhoben.

5 repräsentative Äpfel pro Variante wurden für eine interne Verkostung (Panel von $5 \mathrm{Ex}$ perten) beiseitegelegt, um mögliche störende Aromen, sogenannte "off-flavours", in den Äpfeln zu bestimmen.

\section{UNTERSUCHUNGEN ZUR SORTE}

FUJI

Im Versuchsjahr 2009-2010 wurden Äpfel der Sorte Fuji aus zwei unterschiedlichen Genossenschaften ( $E$ und $L$ ) bei der Anlieferung nach der Ernte ausgesucht und sieben Monate lang in CA gelagert. Nach der Auslagerung $\left(1.3^{\circ} \mathrm{C}\right)$ wurden die Äpfel vor der Wachsbehandlung bei drei unterschiedlichen Lufttemperaturen für 48 Stunden vortemperiert: $1.3^{\circ} \mathrm{C}$ wie in der Praxis, $10^{\circ} \mathrm{C}$ und $20^{\circ} \mathrm{C}$. Nach den 48 Stunden wurden alle Früchte (50 pro Variante) wie in der Praxis für 7 Minuten in $30^{\circ} \mathrm{C}$ warmes Wasser getaucht und anschließend mit Schellack behandelt. Dadurch wollte man einen möglichen Einfluss der Schellack-Behandlung und der verschiedenen Temperaturgradienten auf die Entstehung der Lentizellen-Schwäche (lenticel breakdown) untersuchen. Anschließend wurden die Früchte in Shelf Life bei $20^{\circ} \mathrm{C}$ und $60-70 \%$ r.F. zurückgestellt und 
7 Tage lang täglich visuell ausgewertet. Zudem wurden gesunde und befallene Früchte mit dem DA-Meter (Sinteleia, Bologna, Italien), einem Gerät, das den Chlorophyllgehalt der Früchte nicht-destruktiv bestimmt, gemessen.

Der DA-Meter Wert wurde an jeweils zwei äquatorialen Punkten, einmal auf der Sonnen- und einmal auf der Schattenseite, jeder Frucht gemessen, wobei der Mittelwert dieser beiden Messungen als Bezugswert verwendet wird.

Abschließend wurde nach 7 Tagen Shelf Life der Mineralstoffgehalt von gesunden und befallenen Früchten ermittelt.

\section{ERGEBNISSE}

\section{UNTERSUCHUNGEN ZUR SORTE RED DELICIOUS}

In beiden Versuchsjahren zeigten die Analysen der inneren chemisch-physikalischen Qualitätsparameter nach 6 Monaten Lagerung und 7 Tagen Shelf Life eine deutlich höhere Fruchtfleischfestigkeit (ca. $0.5 \mathrm{~kg} / \mathrm{cm}^{2}$ ) bei den mit Schellack behandelten Früchten im Vergleich zur Kontrolle, sowohl in der ULO-, als auch in der DCA-CF-Lagerung. Die mit SmartFresh (1-MCP) behandelten Früchte zeigten im Vergleich zur Kontrolle hingegen keine signifikanten Unterschiede auf, dies wahrscheinlich deshalb, weil eine Behandlung mit 1-MCP allein schon eine optimale Festigkeitserhaltung garantiert (Abb. 3).

Bezüglich der anderen Qualitätsparameter, wie titrierbare Säure (Abb. 4) und lösliche Trockensubstanz (Abb. 5), konnten keine relevanten Einflüsse der Wachsbehandlung bei den verschiedenen Lagerbedingungen aufgezeigt werden.

Die Auswertung der äußeren Schäden bestätigt die Wirksamkeit der SmartFresh Behandlung (1-MCP) und der DCA-CF Lagerung bei der Kontrolle der Schalenbräuneentwicklung auch nach Shelf Life. Interessanterweise haben die in ULO gelagerten Äpfel, welche einer Schellack Behandlung unterzogen wurden, während der Shelf Life Periode deutlich weniger Schalenbräune entwickelt (Abb. 6), als die ungewachste Variante. Dies wurde vor allem im ersten Versuchsjahr deutlich aufgezeigt.

Im zweiten Versuchsjahr hat sich diese Wirkung auf die Anzahl betroffener Früchte nicht so stark gezeigt wie im Vorjahr, es konnten aber leichte Unterschiede in der Intensität der Schadensausprägung festgestellt werden. Die ungewachsten Äpfel hatten im Vergleich zu den gewachsten einen höheren Anteil an schwer befallenen Früchten $(2.2 \%)$.

Es muss aber auch berücksichtigt werden, dass Früchte im fortgeschrittenen Reifestadium (zweite Pflücke) weniger anfällig für Schalenbräune sind (Abb. 6), und dass die Anfälligkeit stark von den Witterungsbedingungen abhängig ist.

Die Auswertung der inneren Schäden hat keinen deutlichen Zusammenhang zwischen der Schellack-Behandlung und der Entstehung von Physiopathologien bzw. Fäulnis gezeigt. Was beobachtet werden konnte, ist eine deutliche Zunahme der inneren Verbräunungen mit zunehmender Reife, wahrscheinlich durch Seneszenzprozesse verursacht, welche nur durch DCA-CF beinahe vermieden wurden (Abb. 7). Im ersten Versuchsjahr wurden bezüglich äußerer Schäden oder Fäulnis mehr Verluste bei der ungewachsten ULO-Variante, im Vergleich zu den anderen Varianten, beobachtet. Diese Tendenz konnte im zweiten Versuchsjahr nicht bestätigt werden (Abb. 8).

Nach einer Woche Shelf Life wiesen die mit Wachs behandelten Früchte, besonders in der ULO-Variante, einen niedrigeren Gewichtsverlust auf (Abb. 9).

Im Rahmen einer von 5 Experten durchgeführten Verkostung wurde bei einzelnen Früchten ein leichtes Waldbeeren-Aroma festgestellt; einzelne Früchte hatten einen grasigen Geschmack. Diese Unterschiede waren eher auf den Reifezustand der Früchte zurückzuführen als auf die Lagerbedingungen bzw. die Wachsbehandlung, denn diese Aromen traten bei allen Varianten auf. Bei der Verkostung von länger gelagerten Früchten wurde bei den mit Schellack behandelten Früchten eine höhere Knackigkeit festgestellt.

\section{UNTERSUCHUNGEN ZUR SORTE FUJI}

In Zusammenhang mit der Wachsbehandlung wurden in den Obstgenossenschaften vor allem bei langfristig (6 Monate und mehr) gelagerten Partien Symptome einer Physiopathologie, die die Lentizellen befällt, (Lentizellen-Schwäche oder lenticel breakdown) beobachtet.

Es handelt sich dabei um kleine braune Einsenkungen mit einem Durchmesser von
1-8 $\mathrm{mm}$ rund um die Lentizellen, welche meist auf der Schattenseite der Frucht auftreten. Im Anfangsstadium erkennt man eine leichte Einsenkung der Lentizellen; mit zunehmender Reife werden diese größer und tiefer (Abb. 1).

Das darunterliegende Fruchtfleisch ist nicht befallen. Später kann sich sekundäre Fäulnis aufgrund von Infektionen der beschädigten Gewebe entwickeln (Abb. 2).

Laut der am Versuchszentrum durchgeführten Untersuchung scheint die Schellack-Behandlung sowohl bei $1.3{ }^{\circ} \mathrm{C}$ als auch bei $20{ }^{\circ} \mathrm{C}$ Vortemperierung nichts auszulösen. Der Schaden scheint alleine durch die tiefen Temperaturen von $1.3{ }^{\circ} \mathrm{C}$ ausgelöst zu werden (Abb. 10). Die Untersuchung des Entwicklungsverlaufes des Lentizellen-Zusammenbruchs (Abb. 10 bis) bestätigt diese Beobachtung. Bei $20{ }^{\circ} \mathrm{C}$ wiesen die behandelten Früchte keine Schäden auf, die unbehandelten nur ein leichtes Ausmaß. Es wurde nur ein leichter Anstieg der beschädigten Früchte während der 7 Tage Shelf Life bei Raumtemperatur beobachtet, wie der Vergleich nach 1 Tag (Abb. 11) mit 7 Tagen Shelf Life (Abb. 12) zeigt. Die Schadensintensität und die Entstehung von sekundären Infektionen, welche Fäulnis verursachen, nahm hingegen zu.

Das Aufwärmen der kalten Früchte $\left(1.3^{\circ} \mathrm{C}\right)$ auf $20{ }^{\circ} \mathrm{C}$, vor dem Eintauchen in $30^{\circ} \mathrm{C}$ warmes Wasser, hat hingegen einen positiven Einfluss bezüglich der Vorbeugung dieser Physiopathologie gezeigt. Auch das Temperieren auf $10{ }^{\circ} \mathrm{C}$ bestätigt die Wichtigkeit der langsamen Erwärmung, um einen Temperaturschock zu vermeiden (Abb. 11)

Das direkte Eintauchen der kalten Früchte $\left(1.3^{\circ} \mathrm{C}\right)$ ohne vorheriger Erwärmung in $30^{\circ} \mathrm{C}$ warmes Wasser für 7 Minuten hat die Epidermis auf eine Temperatur zwischen 18 $20{ }^{\circ} \mathrm{C}$ erwärmt. Messungen der Temperatur im Kernfruchtfleisch mit einer Temperatursonde ergaben einen Temperaturgradienten zur Epidermis von $6-7^{\circ} \mathrm{C}$. Diese Bedingungen haben die Entwicklung der Lentizellen-Schwäche begünstigt (Abb. 11 und Abb. 12).

Es konnte keine signifikante Korrelation zwischen dem anhand des DA-Indexes geschätzten Reifezustand der ausgelagerten Äpfel und dem Auftreten des Schadens aufgezeigt werden. Die DA-Werte von beschädigten und gesunden Früchten waren beinahe identisch, sodass das Auftreten des Schadens nicht auf eine Überreife der 
Früchte zurückgeführt werden kann (DAMittelwert: 1 für Genossenschaft E und 0.8 für Genossenschaft L).

Der Mineralstoffgehalt der gesunden, im Vergleich zu den durch den Lentizellen-Zusammenbruch schwer beschädigten Früchten, zeigte nur in zwei von vier Fällen Unterschiede im K/Ca und N/Ca Verhältnis, welche allerdings innerhalb des Normbereichs bezüglich Lagerstippe lagen (Abb. 13 und Abb. 14).

\section{SCHLUSSFOLGERUNG}

\section{UNTERSUCHUNGEN ZUR SORTE RED DELICIOUS}

Als Ergebnis unserer zweijährigen Versuchstätigkeit zur Prüfung der Nacherntebehandlung mit einem Wachsprodukt auf Basis von Schellack (Xedafos $L$ ) haben die behandelten Früchte nicht nur ein besseres Aussehen erlangt, sondern auch eine Verlängerung der Shelf Life Phase durch eine erhöhte Fruchtfleischfestigkeit und einen niedrigeren Gewichtsverlust, bedingt durch die verringerte Wassertranspiration und Gasdiffusion.

Außerdem hat die Wachsbehandlung keinen Einfluss auf die Entstehung von inneren Verbräunungen gezeigt. Spät geerntete Früchte waren hingegen anfälliger gegenüber dieser Physiopathologie. Ein wichtiger Aspekt, der noch weiter untersucht werden sollte, ist der Einfluss der Wachsbehandlung auf die Entwicklung der Schalenbräune nach Shelf Life unter ULO-Lagerungsbedingungen. Im ersten Versuchsjahr war tatsächlich der Anteil von betroffenen Äpfeln, im Vergleich zu den nicht behandelten Äpfeln, in der Schellack-Variante viel niedriger. Im zweiten Versuchsjahr hat sich dieser große Unterschied nicht mehr gezeigt, allerdings waren die Äpfel generell weniger anfällig.

Der Schalenbräune wurde erfolgreich mittels 1-MCP-Behandlung (SmartFresh) oder DCA-CF-Lagerung auch während Shelf Life entgegengewirkt.

Die Wachsbehandlung hatte keinen Einfluss auf die Entstehung von Fäulnis.

Bei der von einem Panel durchgeführten Verkostung haben sich keine deutlichen Unterschiede in Bezug auf Aromen- bzw. Störaromabildung bei den verschiedenen Lagerbedingungen und Behandlungen gezeigt.

\section{ZUSAMMENFASSUNG}

Die Nachernte-Beschichtung von Früchten mit Schellack verbessert deren Aussehen. Zudem könnte die innere Qualität besser erhalten und physiologischen Störungen, wie z.B. der gewöhnliche Schalenbräune, vorgebeugt werden. Während eines zweijährigen Versuches wurde im Versuchszentrum Laimburg der Einfluss einer Schellack-Behandlung an den Sorten Red Delicious und Fuji bei unterschiedlichen Varianten überprüft. Ausgewertet wurden die Qualitätsunterschiede zwischen gewachsten und ungewachsten Früchten nach einer Lagerung in CA-ULO, CA-ULO mit 1-MCP (SmartFresh) oder in dynamisch kontrollierter Atmosphäre mittels Chlorophyll-Fluoreszenz-Sensoren (DCA-CF). Bei gewachsten Früchten der Sorte Red Delicious konnte nach 7 Tagen Shelf Life neben einer höheren Festigkeit auch eine Hemmung des Wasserverlustes und der gewöhnlichen Schalenbräune beobachtet werden. Bei behandelten Früchten der Sorte Fuji konnte bestätigt werden, dass die Lentizellen-Schwäche (lenticel breakdown) auf den Temperaturschock während der Wachsbehandlung zurückzuführen ist und nicht auf die Behandlung selbst.

\section{RIASSUNTO}

L'operazione di ceratura con gommalacca naturale oltre a migliorare l'aspetto dei frutti trattati, può migliorarne la qualità intrinseca e talora prevenire anche disordini di tipo fisiologico come il riscaldo superficiale. Nei due anni di indagine presso il Centro di Sperimentazione Laimburg è stata studiata l'influenza dell'applicazione di gommalacca su diverse tesi, considerando perciò l'influenza sulla qualità con e senza ceratura, eseguita dopo conservazione in CA-ULO, CA-ULO con 1-MCP (SmartFresh) o CA dinamica tramite sensori della fluorescenza della clorofilla (DCA-CF). Su Red Delicious trattate con la cera dopo 7 giorni di shelf life è stato osservato un trend nella ridotta evapotraspirazione e nella minore suscettibilità al riscaldo superficiale, oltre ad una maggiore durezza della polpa. Su Fuji trattate abbiamo potuto dimostrare che il danno denominato disfacimento lenticellare (lenticel breakdown) era causato dallo shock termico dovuto al processo di ceratura e non alla ceratura stessa.

\section{UNTERSUCHUNGEN ZUR SORTE FUJI}

Nach den zahlreichen Beobachtungen aus der Praxis bezüglich 'lenticel breakdown' (Lentizellen-Schwäche), welche erst nach der Schellack-Behandlung ausgelagerter Partien auftrat, haben die gewonnenen Ergebnisse gezeigt, dass ein eindeutiger Zusammenhang zwischen der Schadensentstehung und dem Temperaturschock, dem die kalten Früchte aufgrund des Eintauchens ins wärmere Wasser unterworfen waren, besteht.

Zudem konnten wir die in Washington State gemachten Beobachtungen (Kupferman, 2009) [3] bestätigen, dass Früchte mit unausgeglichenem Mineralstoffgehalt anfälli- ger gegenüber der erwähnten Physiopathologie sein können. Die am meisten befallenen Sorten sind Gala und Fuji, aber der Lentizellen-Zusammenbruch wurde in den USA auch für andere Sorten, wie Granny Smith, Golden Delicious und Cripps Pink aufgezeigt (Washington State; Curry, 2003) [4]. Das trockene Klima in Zusammenhang mit der raschen Fruchtentwicklung spielt sicher eine wichtige Rolle bezüglich der Kutikula- und Lentizellen-Struktur (Turketti et al., 2012) [5]. Tatsächlich wurde ein vorbeugender Effekt durch die Spritzung von ölhaltigen Emulsionen im Feld kurz vor der Ernte beobachtet (Curry et al., 2008) [6]. Eine andere Ursache spielt das fortgeschrittene Reifestadium (Lotze und Theron, 2010) [7] bzw. die langfristige Lagerung (Kupferman, 2007) [8]. 
Es ist immer ratsam, die Ernte innerhalb des vorgegebenen Erntefensters abzuschließen, eine korrekte Düngung durchzuführen und für einen gleichmäßigen Behang (Blatt/Frucht Verhältnis) durch gezielten Baumschnitt und Ausdünnung zu sorgen.

Als Schlussfolgerung ist die Nacherntebehandlung mit Schellack sicher für jene Märkte interessant, in denen eine konti-

\section{LITERATUR}

[1] Zanella A., [et. al.] (2005). Fruit fluorescence response to low oxygen stress: Modern storage technologies compared to 1-MCP treatment of apple. Acta Horticulturae 682: 15351542.

[2] Kupferman E. (2005). A status report on lenticel breakdown of Gala apples - July 2005.

Postharvest Information Network. http://postharvest.tfrec.wsu.edu/ EMK2005A.pdf.

[3] Kupferman E. (2009). Lenticel breakdown of apples \& fruit mineral balance. Postharvest Information Network. http://postharvest.tfrec.wsu.edu/EMK2009A. nuierliche Kühlkette nicht immer gewährleistet werden kann, oder bei kritischen Shelf Life Bedingungen.

\section{DANKSAGUNG}

Wir möchten uns für die exzellente Mitarbeit von Paul Cazzanelli und für die ausgezeichnete Zusammenarbeit mit Oswald

[4] Curry E. (2003). Factors associated with apple lenticel breakdown. Postharvest Information Network.

http://postharvest.tfrec.wsu.edu/ REP2003B.pdf.

[5] Turketti S.S., Curry E., Lotze E. (2012). Role of lenticel morphology, frequency and density on incidence of lenticel breakdown in 'Gala' apples. Scientia Horticulturae 138: 90-95.

[6] Curry E., Torres C., Neubauer L. (2008). Preharvest lipophilic coatings reduce lenticel breakdown disorder in 'Gala' apples. HortTechnology 18: 4, 690-696.
Rossi, Ines Ebner und Aldo Matteazzi vom Versuchszentrum Laimburg bedanken; bedanken möchten wir uns auch bei Gerhard Dichgans und Hannes Moser für den Kontakt mit den Obsgenossenschaften des VOG Südtirol.

[7] Lotze E., Theron K.I. (2009/2010). Investigating factors contributing to the development of lenticel breakdown in 'Royal Gala' apples in South Africa. SA Fruit Journal 8: 6, 67-68.

[8] Kupferman E. (2007). Plain talk about apple lenticel breakdown. http://postharvest.tfrec.wsu.edu/EMK2007C.pdf. 


\section{ANHANG: ABBILDUNGEN}

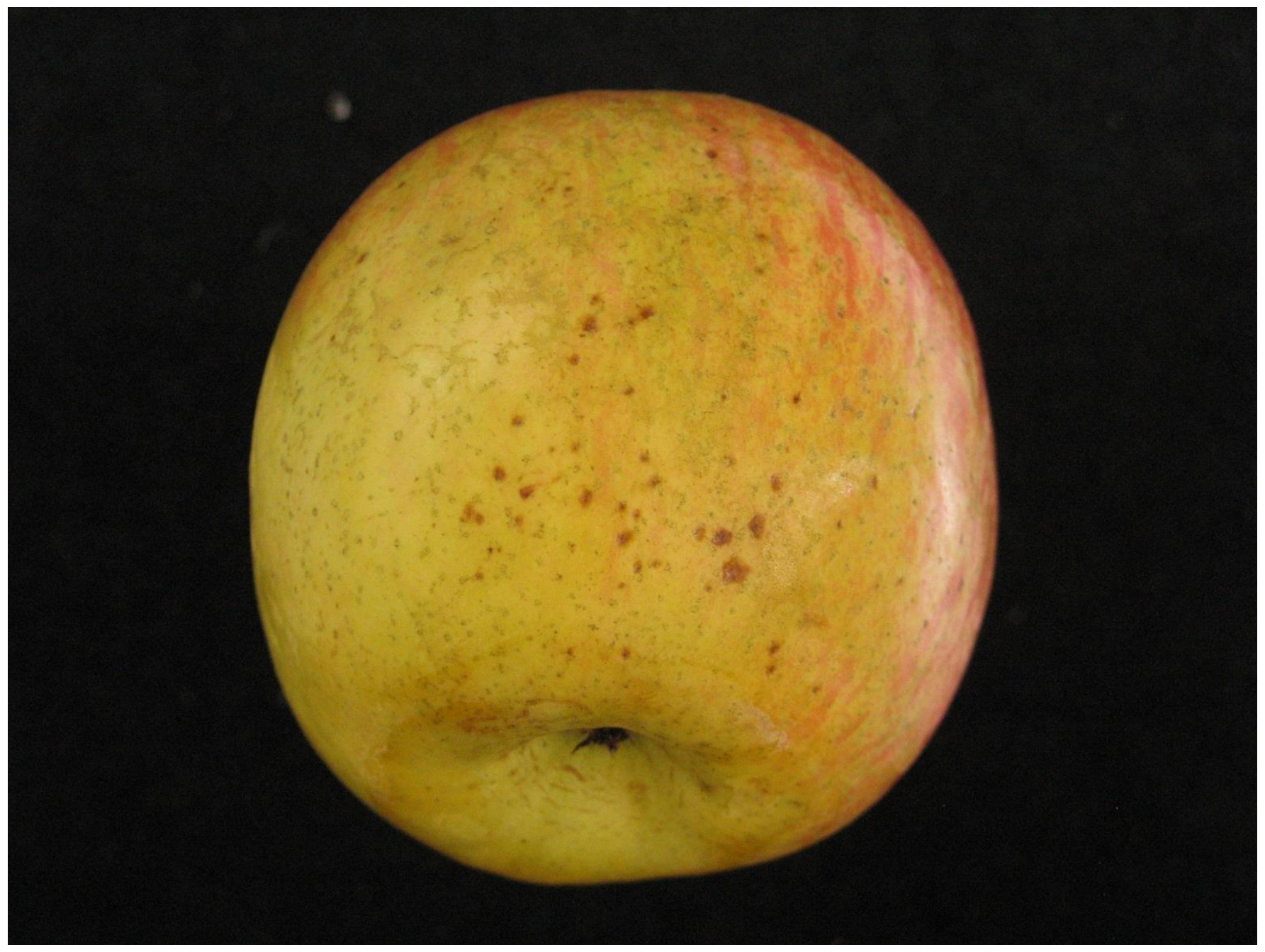

Abb. 1: Lentizellen-Schwäche (lenticel breakdown) auf Fuji. // Lenticel Breakdown on Fuji. 


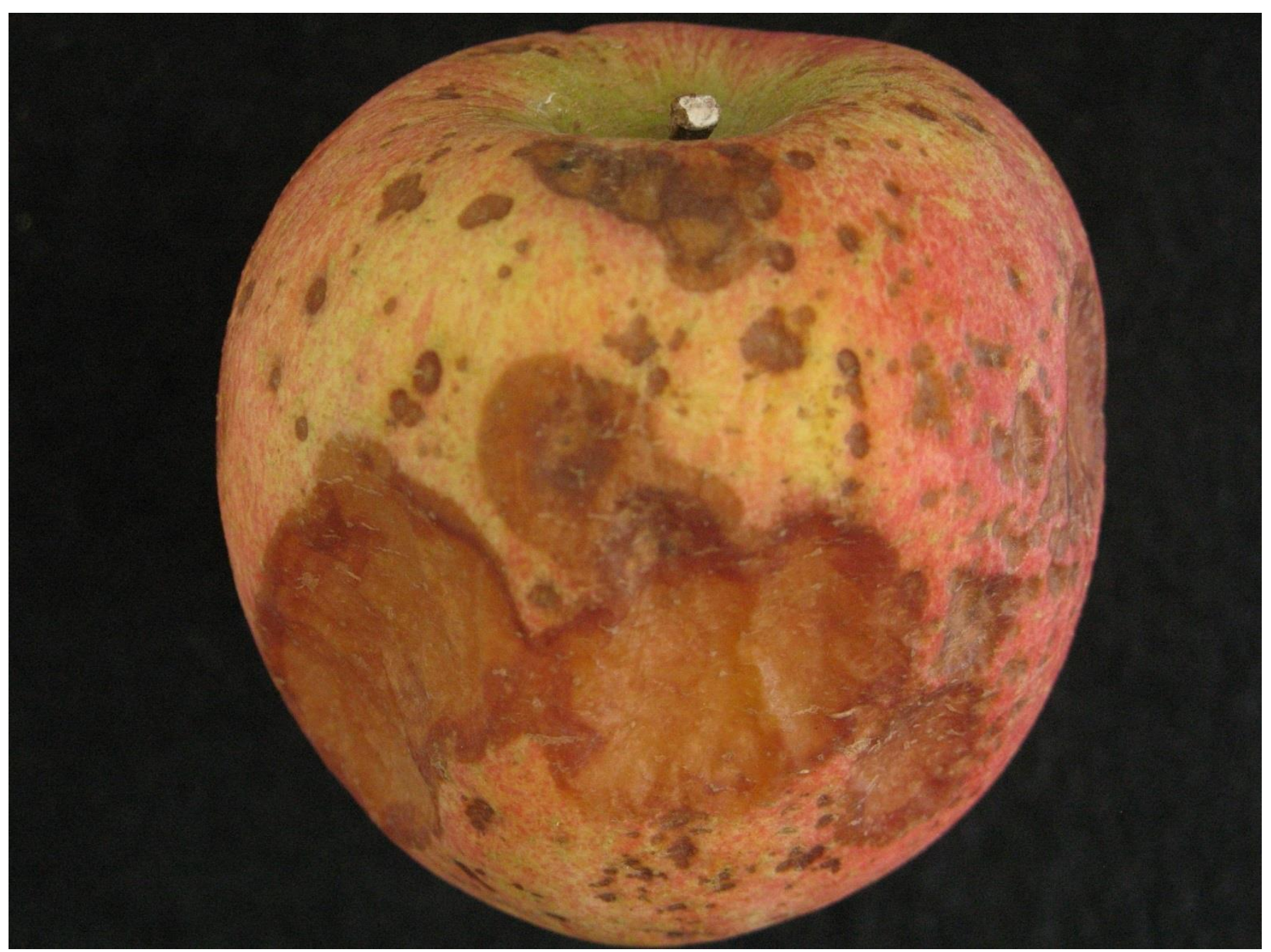

Abb. 2: Lentizellen-Schwäche und schwere Symptome von sekundärer Fäulnis auf Fuji. // Lenticel Breakdown and severe symptoms of secondary rots on Fuji. 

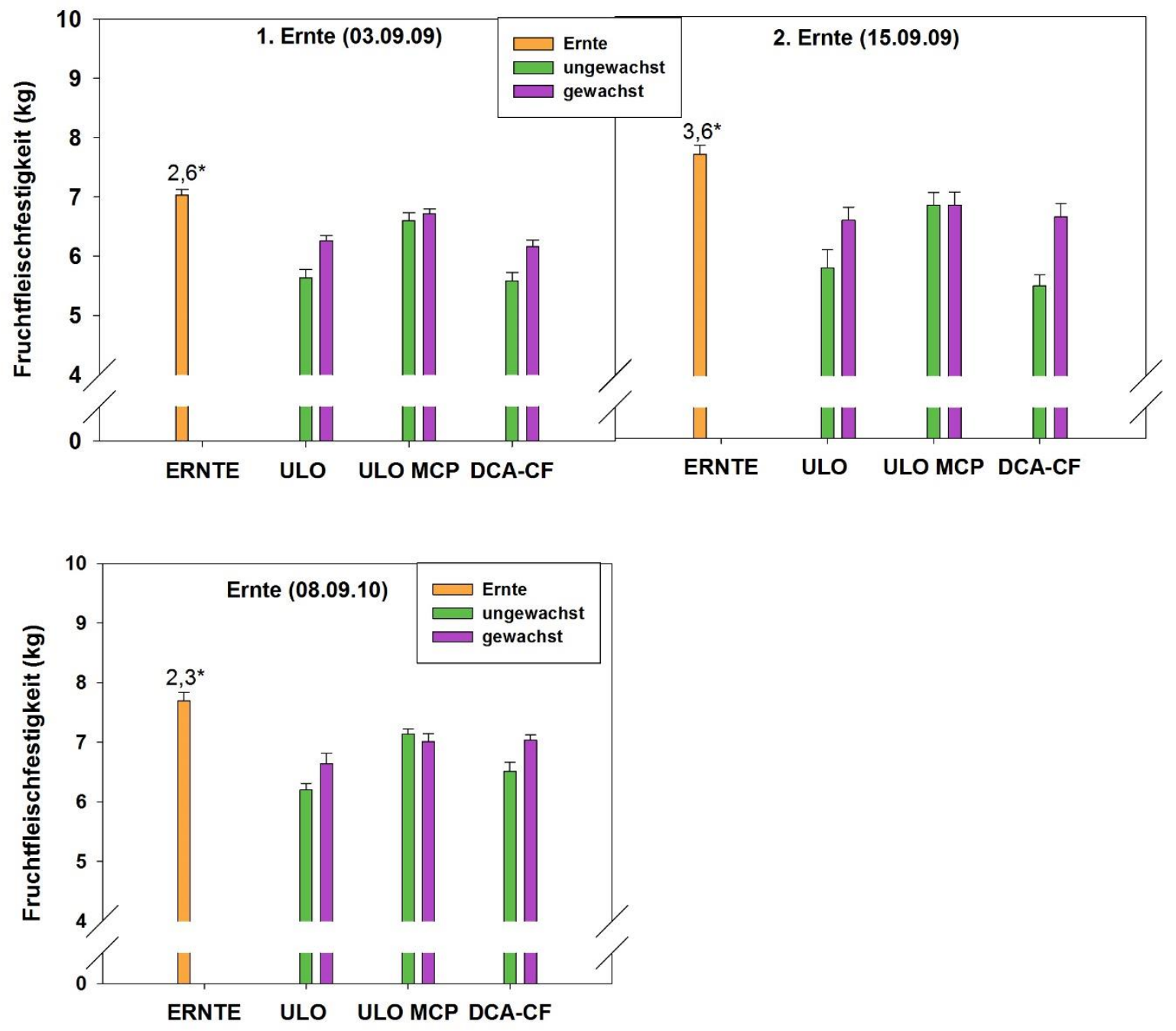

Der Fehlerbalken entspricht den Standardfehler - *Stärkewert (Laimburg Skala 1-5)

Abb. 3: Einfluss der Schellack-Behandlung auf die Fruchtfleischfestigkeit der Sorte Red Delicious (1. und 2. Ernte der Saison 2009-2010 und einzige Ernte 2010-2011) nach 6 Monaten Lagerung (ULO, ULO +1-MCP, DCA-CF) und 7 Tagen Shelf Life. // Shellac treatment influence on the firmness of Red Delicious variety (1st and 2nd harvest in the season 2009-2010 and single harvest in the season 2010-2011) after 6 months storage (ULO, ULO $+1-M C P, D C A-C F)$ and 7 days shelf life. 

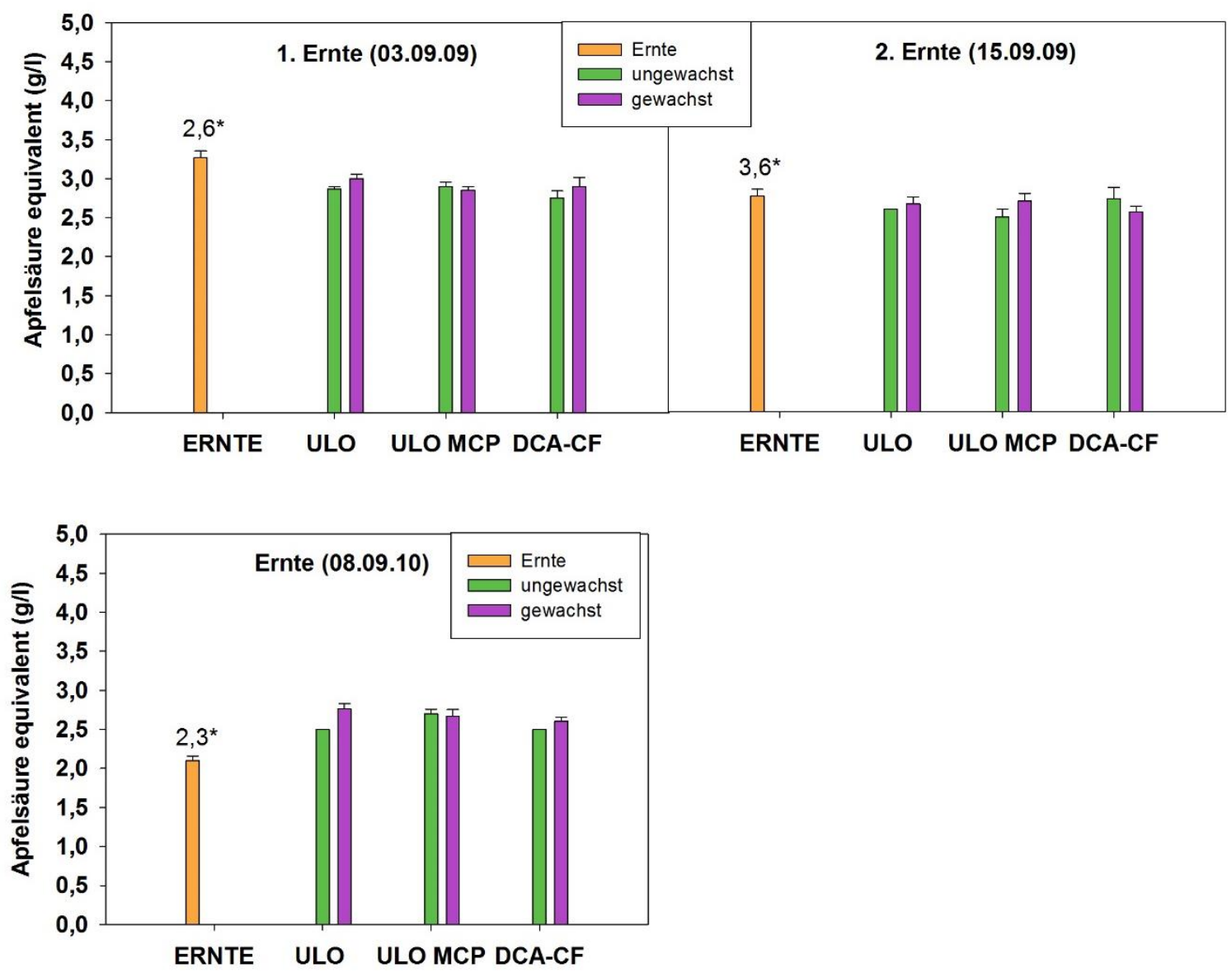

Der Fehlerbalken entspricht den Standardfehler - *Stärkewert (Laimburg Skala 1-5)

Abb. 4: Einfluss der Schellack-Behandlung auf den Säuregehalt der Sorte Red Delicious (1. und 2. Ernte der Saison 2009-2010 und einzige Ernte 20102011) nach 6 Monaten Lagerung (ULO, ULO +1-MCP, DCA-CF) und 7 Tagen Shelf Life. // Shellac treatment influence on the acid content of Red Delicious variety (1st and 2nd harvest in the season 2009-2010 and single harvest in the season 2010-2011) after 6 months storage (ULO, ULO +1$M C P, D C A-C F)$ and 7 days shelf life. 

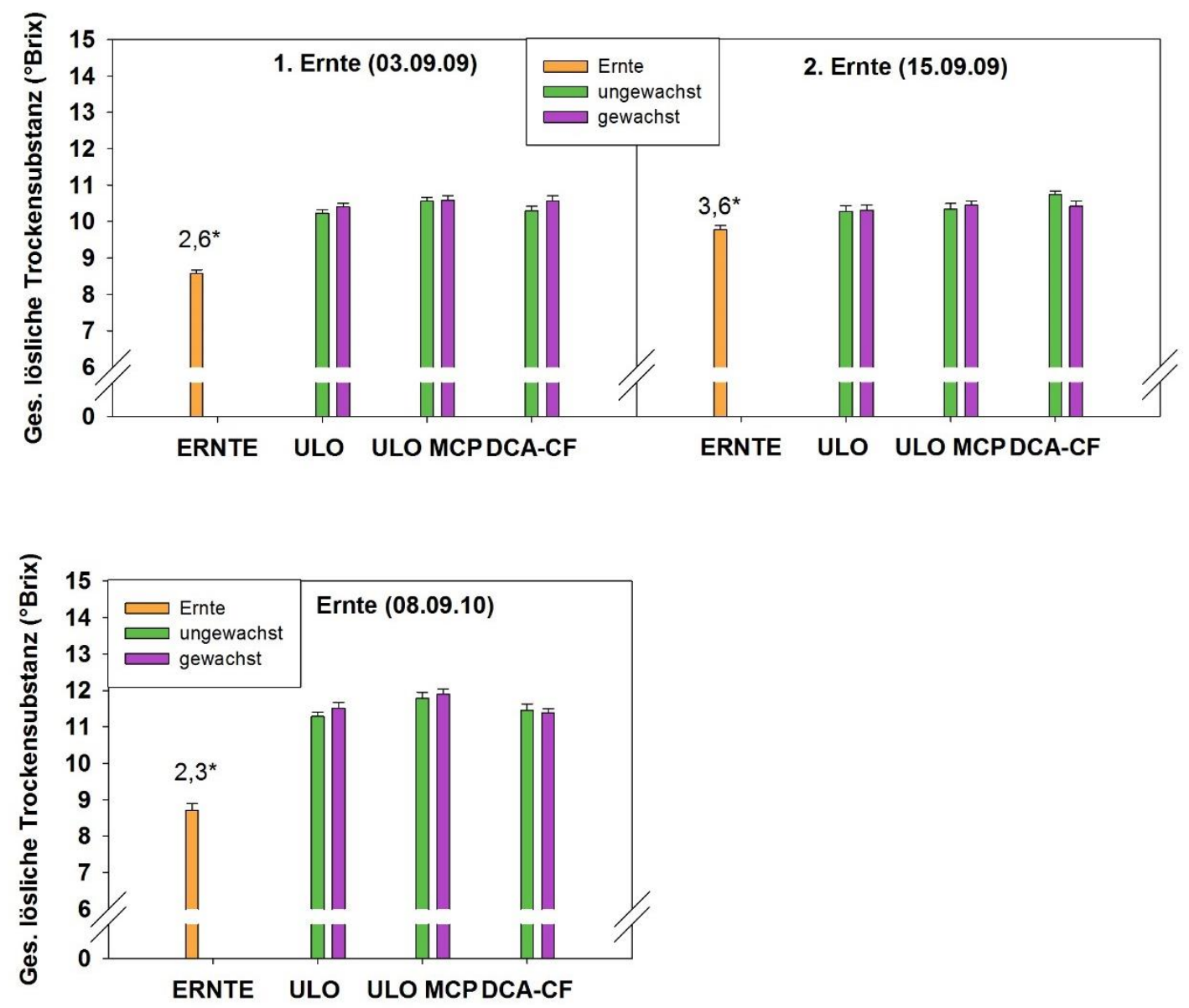

Der Fehlerbalken entspricht den Standardfehler - *Stärkewert (Laimburg Skala 1-5)

Abb. 5: Einfluss der Schellack-Behandlung auf den Zuckergehalt der Sorte Red Delicious (1. und 2. Ernte der Saison 2009-2010 und einzige Ernte 2010-2011) nach 6 Monaten Lagerung (ULO, ULO +1-MCP, DCA-CF) und 7 Tagen Shelf Life. // Shellac treatment influence on the sugar content of Red Delicious variety (1st and 2nd harvest in the season 2009-2010 and single harvest in the season 2010-2011) after 6 months storage (ULO, ULO $+1-M C P, D C A-C F)$ and 7 days shelf life. 

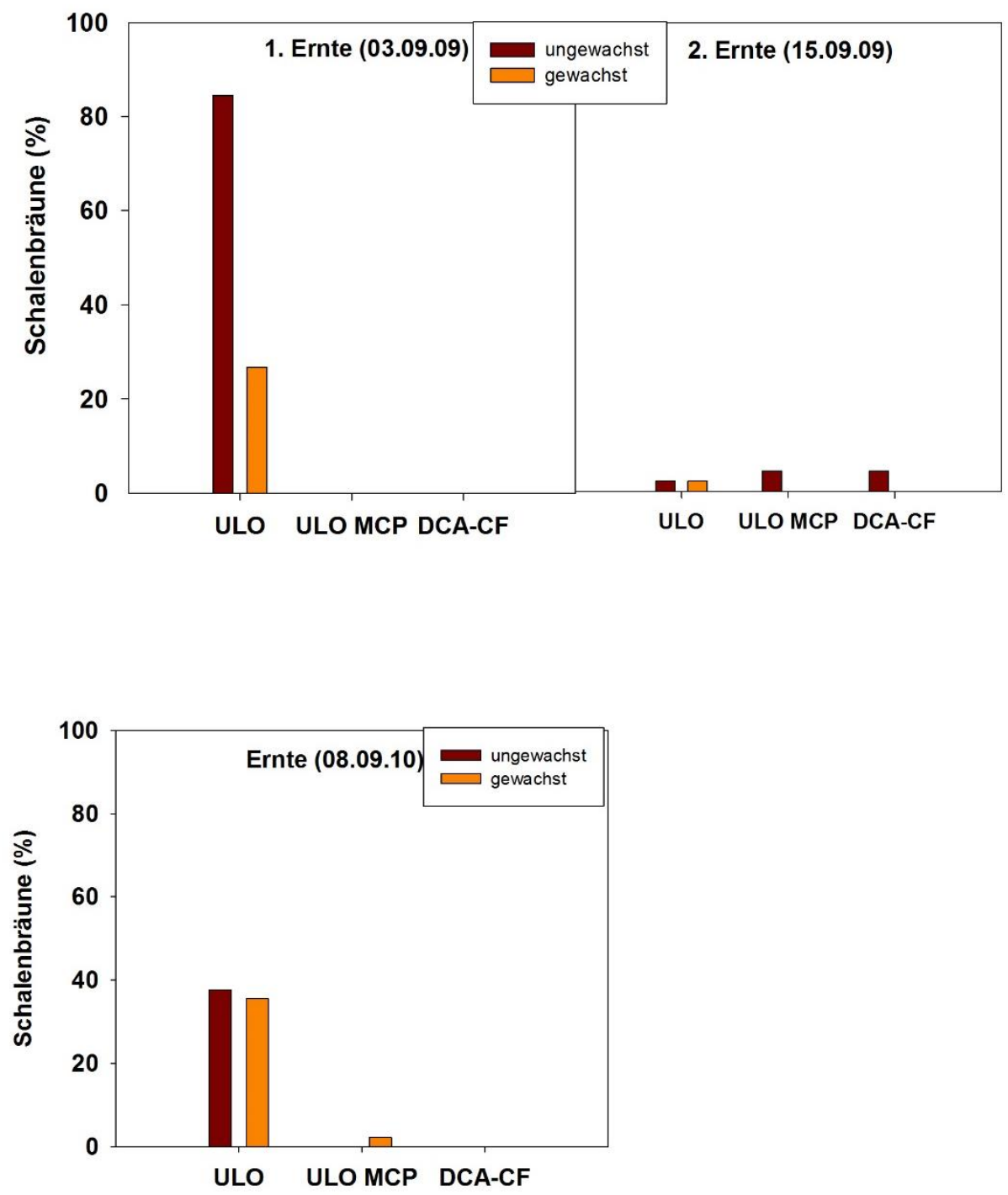

Abb. 6: Einfluss der Schellack-Behandlung auf die Schalenbräune-Anfälligkeit der Sorte Red Delicious (1. und 2. Ernte der Saison $2009-2010$ und einzige Ernte 2010-2011) nach 6 Monaten Lagerung (ULO, ULO +1-MCP, DCA-CF) und 7 Tagen Shelf Life. // Shellac treatment influence on the susceptibility to superficial scald of Red Delicious variety (1st and 2nd harvest in the season 2009-2010 and single harvest in the season 2010-2011) after 6 months storage (ULO, ULO +1-MCP, DCA-CF) and 7 days shelf life. 

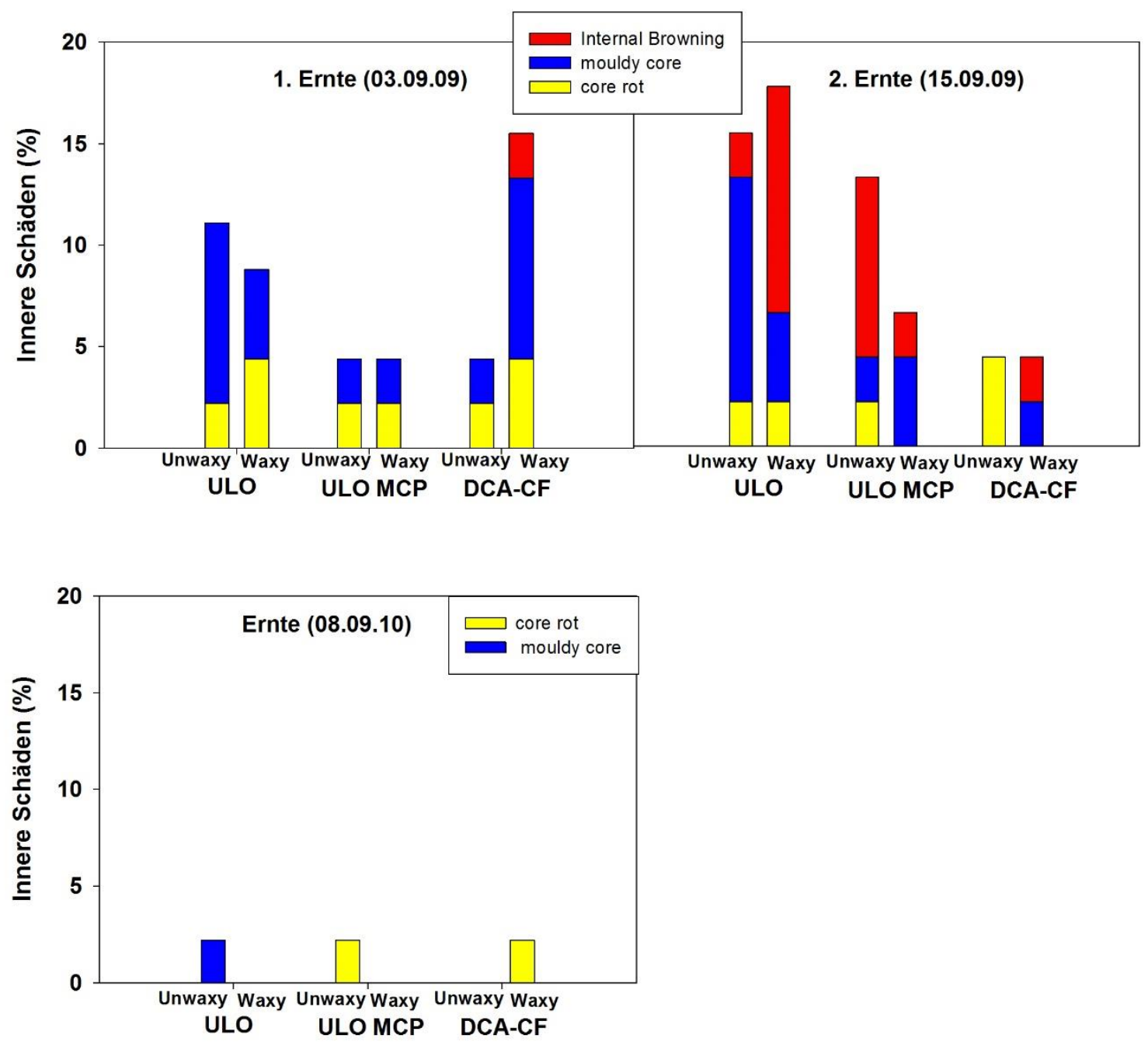

Abb. 7: Einfluss der Schellack-Behandlung auf das Vorkommen von inneren Schäden bei der Sorte Red Delicious (1. und 2. Ernte der Saison 20092010 und einzige Ernte 2010-2011) nach 6 Monaten Lagerung (ULO, ULO +1-MCP, DCA-CF) und 7 Tagen Shelf Life. // Shellac treatment influence on the occurrence of internal damages of Red Delicious variety (1st and 2nd harvest in the season 2009-2010 and single harvest in the season 20102011) after 6 months storage (ULO, ULO +1-MCP, DCA-CF) and 7 days shelf life. 

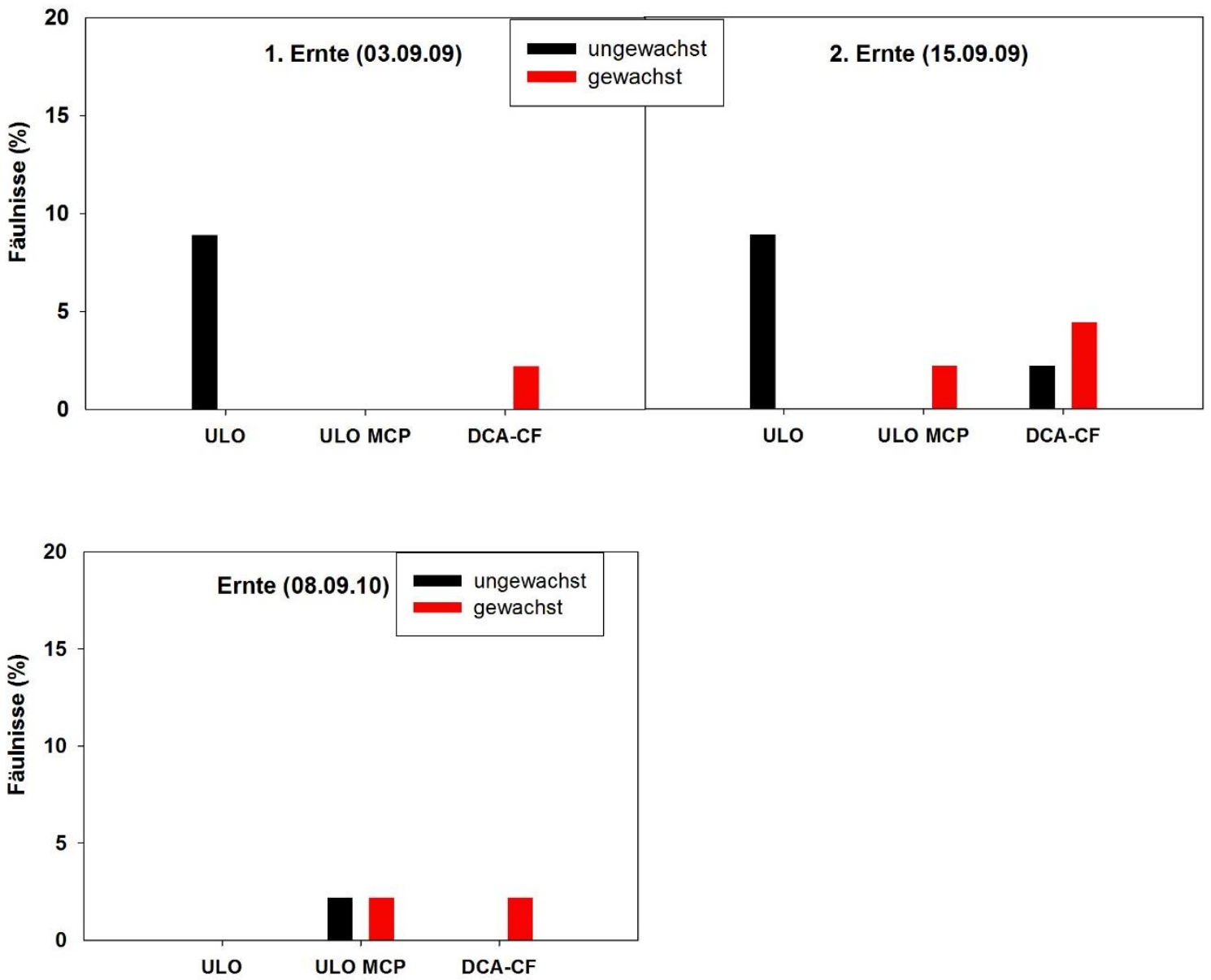

Abb. 8: Einfluss der Schellack-Behandlung auf das Vorkommen von Fäulnis bei der Sorte Red Delicious (1. und 2. Ernte der Saison 2009-2010 und einzige Ernte im 2010-2011) nach 6 Monaten Lagerung (ULO, ULO +1-MCP, DCA-CF) und 7 Tagen Shelf Life. // Shellac treatment influence on rots occurrence of Red Delicious variety (1st and 2nd harvest in the season 2009-10 and single harvest in the season 2010-11) after 6 months storage (ULO, ULO +1-MCP, DCA-CF) and 7 days shelf life. 

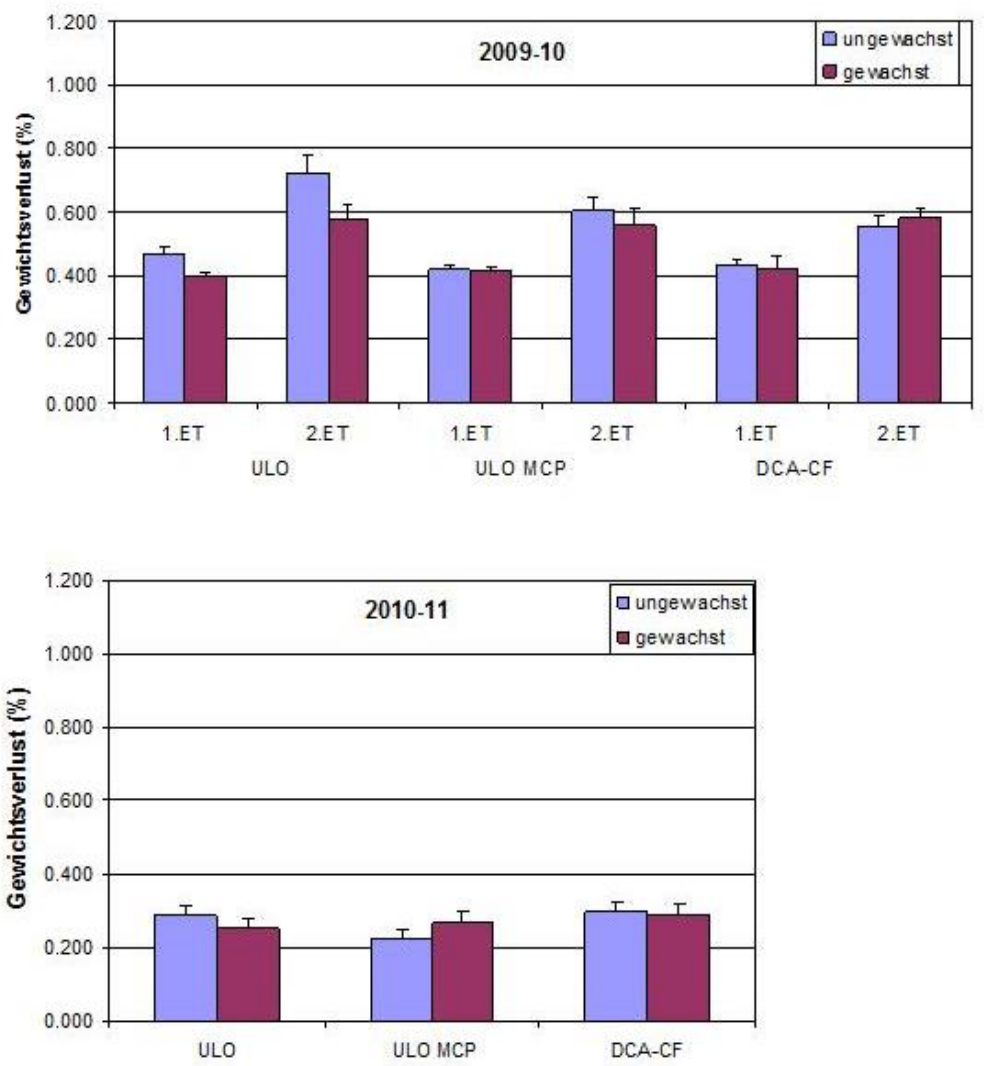

Abb. 9: Einfluss der Schellack-Behandlung auf den Gewichtsverlust bei der Sorte Red Delicious während 7 Tage Shelf Life (1. und 2. Ernte in der Saison 2009-2010 und einzige Ernte in der Saison 2010-2011) nach 6 Monaten Lagerung (ULO, ULO + 1-MCP, DCA-CF). Der Fehlerbalken entspricht dem Standardfehler. // Shellac treatment influence on weight loss of Red Delicious variety during 7 days of shelf life (1st and 2 nd harvest in the season 2009-2010 and single harvest in the season 2010-2011) after 6 months storage (ULO, ULO+1-MCP, DCA-CF). The error bar corresponds to the standard error 


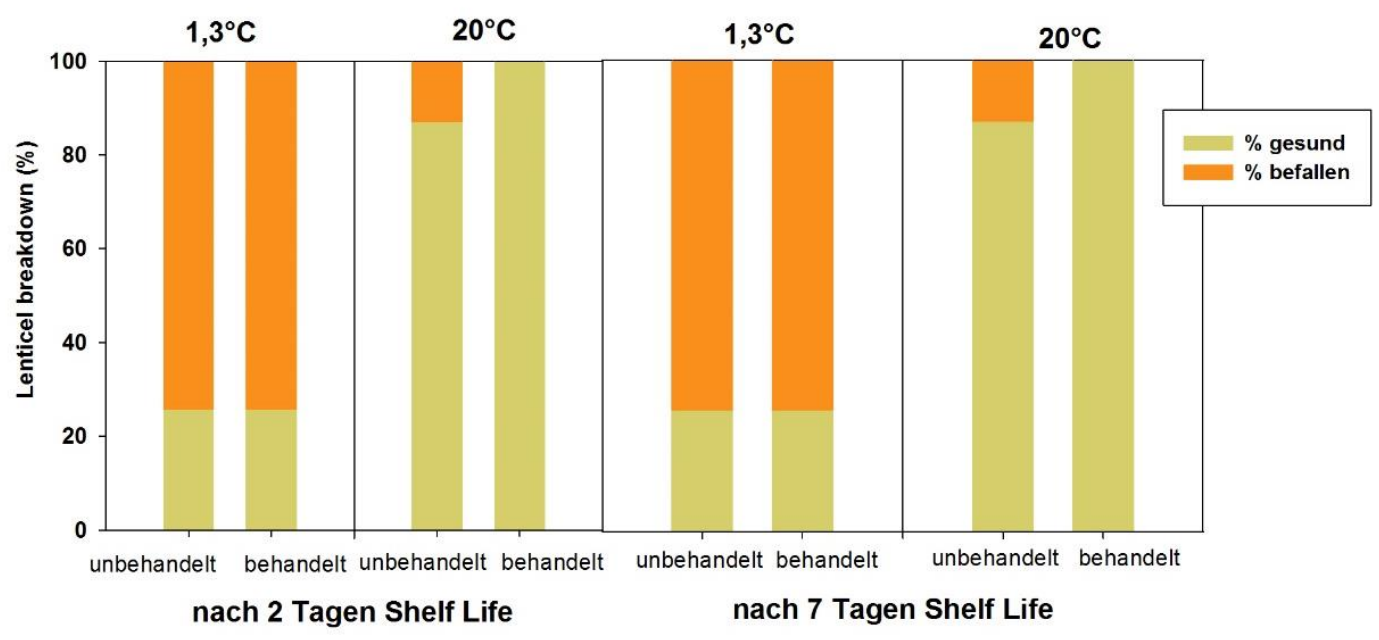

Abb. 10: Anteil gesunder und durch Lentizellen-Zusammenbruch betroffener Früchte der Sorte Fuji (Genossenschaft E) nach Schellack-Behandlung bei unterschiedlicher Vor-Temperierung $\left(1.3^{\circ} \mathrm{C} ; 20^{\circ} \mathrm{C}\right)$, bewertet nach 2 und 7 Tagen Shelf Life. // Healthy and due to 'Lenticel Breakdown' damaged fruits of 'Fuji' variety (cooperative E) after shellac treatment at different pre-tempering $\left(1.3^{\circ} \mathrm{C} ; 20^{\circ} \mathrm{C}\right.$ ), assessed after 2 and 7 days shelf life.
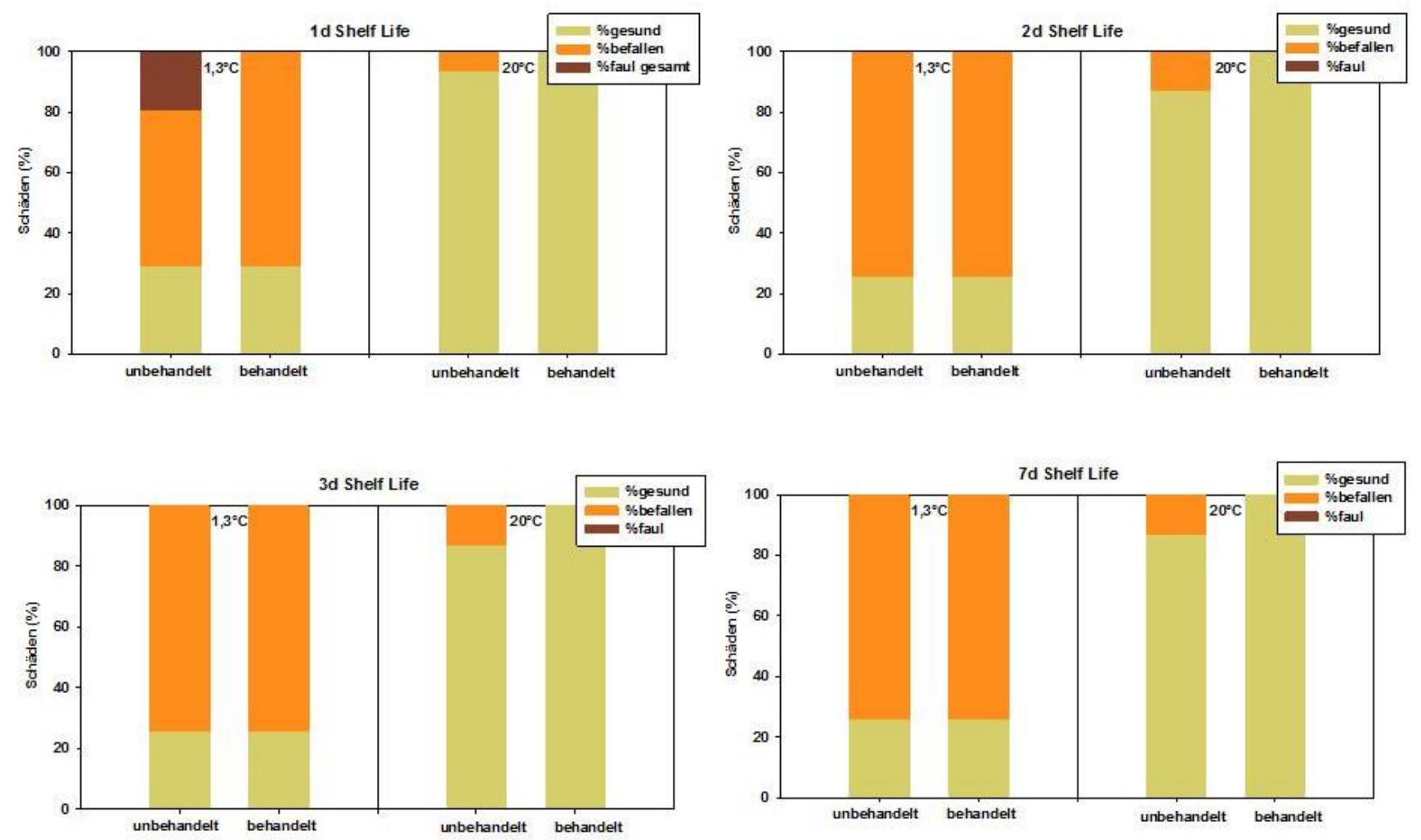

Abb. 10 bis: Verlauf des Lentizellen-Zusammenbruchs (vom 25.05.2010 bis 01.06.2010) mit Anteil gesunder und durch Lentizellen-Zusammenbruch befallener Früchte der Sorte Fuji (Genossenschaft E) nach Schellack-Behandlung bei unterschiedlicher Vor-Temperierung $\left(1.3^{\circ} \mathrm{C} ; 20^{\circ} \mathrm{C}\right) / /$ Evolution of Lenticel Breakdown (from 25/05/2010 to 01/06/2010) splitted between healthy and damaged fruits of cv. Fuji (cooperative E) after shellac treatment at different temperature conditionings $\left(1.3^{\circ} \mathrm{C} ; 20^{\circ} \mathrm{C}\right)$. 


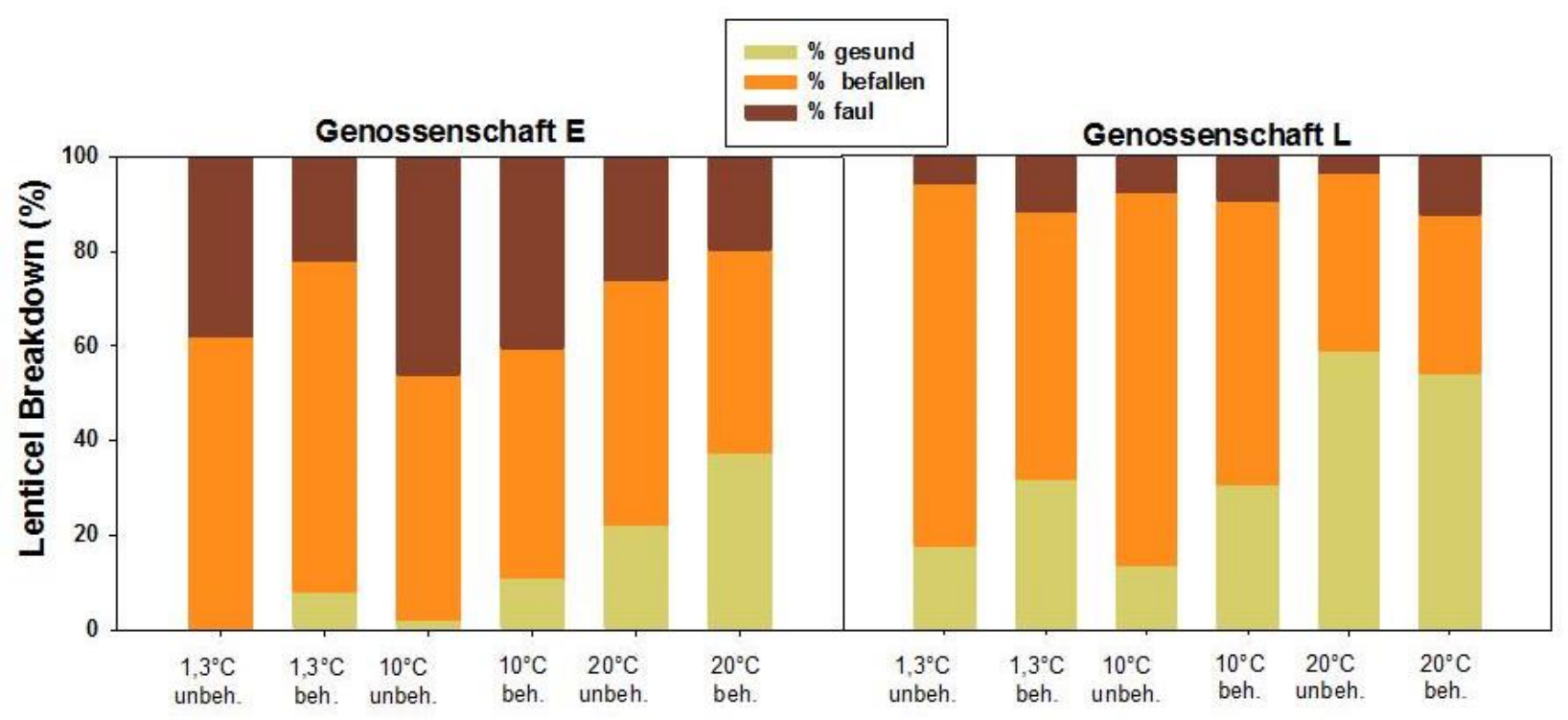

Abb. 11: Anteil gesunder und durch Lentizellen-Zusammenbruch befallener Früchte der Sorte Fuji (Genossenschaften E und L) nach einem Tag Shelf Life nach Schellack-Behandlung bei unterschiedlicher Vor-Temperierung $\left(1.3^{\circ} \mathrm{C} ; 10^{\circ} \mathrm{C} ; 20^{\circ} \mathrm{C}\right.$; unbeh. = unbehandelt; beh. = behandelt). $/ /$ Healthy and due to 'Lenticel Breakdown' damaged fruits of Fuji variety (cooperatives $E$ and $L$ ) after one day shelf life following the shel-lac treatment at different pre-tempering $\left(1.3^{\circ} \mathrm{C} ; 10^{\circ} \mathrm{C} ; 20^{\circ} \mathrm{C}\right.$; unbeh. $=$ control; beh. $=$ treated $)$.

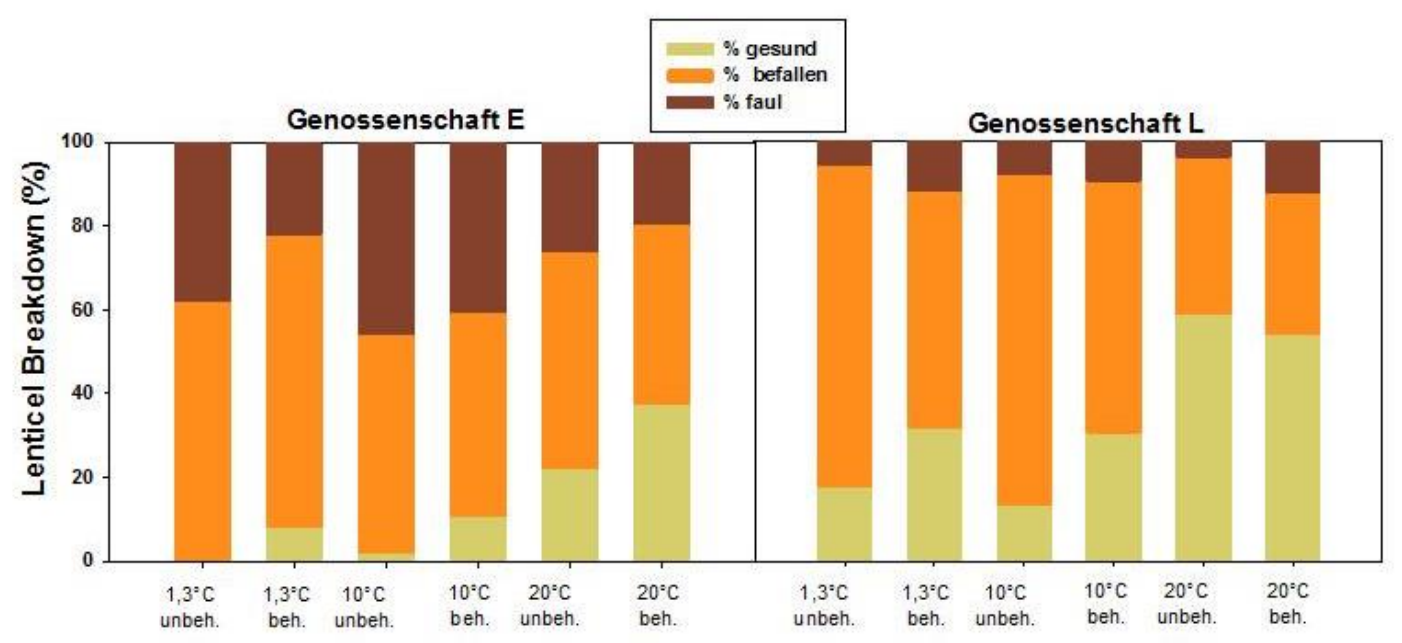

Abb. 12: Anteil gesunder und durch Lentizellen-Zusammenbruch befallener Früchte der Sorte Fuji (Genossenschaften E und L) nach 7 Tagen Shelf Life nach Schellack-Behandlung bei unterschiedlicher Vor-Temperierung $\left(1.3^{\circ} \mathrm{C} ; 10^{\circ} \mathrm{C} ; 20^{\circ} \mathrm{C}\right.$; unbeh. = unbehandelt; beh. = behandelt $) . / /$ Healthy and due to 'Lenticel Breakdown' damaged fruits of Fuji variety (cooperatives $E$ and $L$ ) after 7 days shelf life following the shellac treatment at different pre-tempering $\left(1.3^{\circ} \mathrm{C} ; 10^{\circ} \mathrm{C} ; 20^{\circ} \mathrm{C}\right.$; unbeh. $=$ control; beh. $=$ treated $)$. 


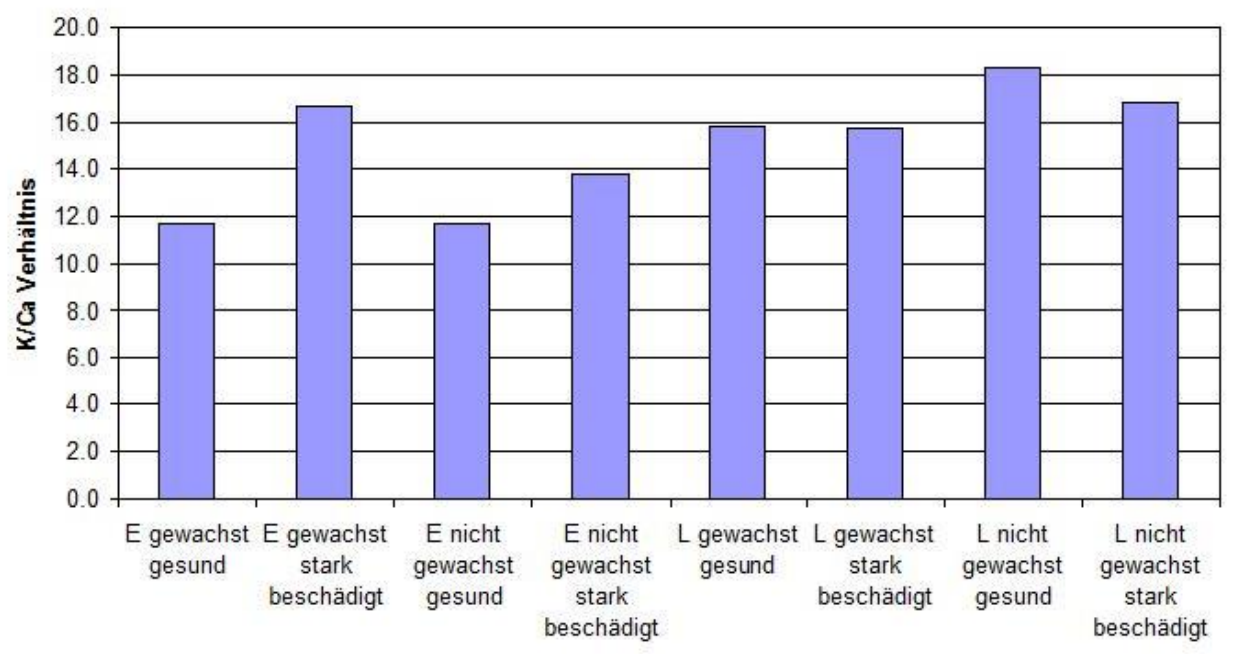

Abb. 13: K/Ca Verhältnis in verschiedenen Herkünften: Vergleich von gesunden und mit Lentizellen-Schwäche befallenen Früchten. // K/Ca ratio of different origins: comparison of healthy and due to 'Lenticel Breakdown' damaged fruits.

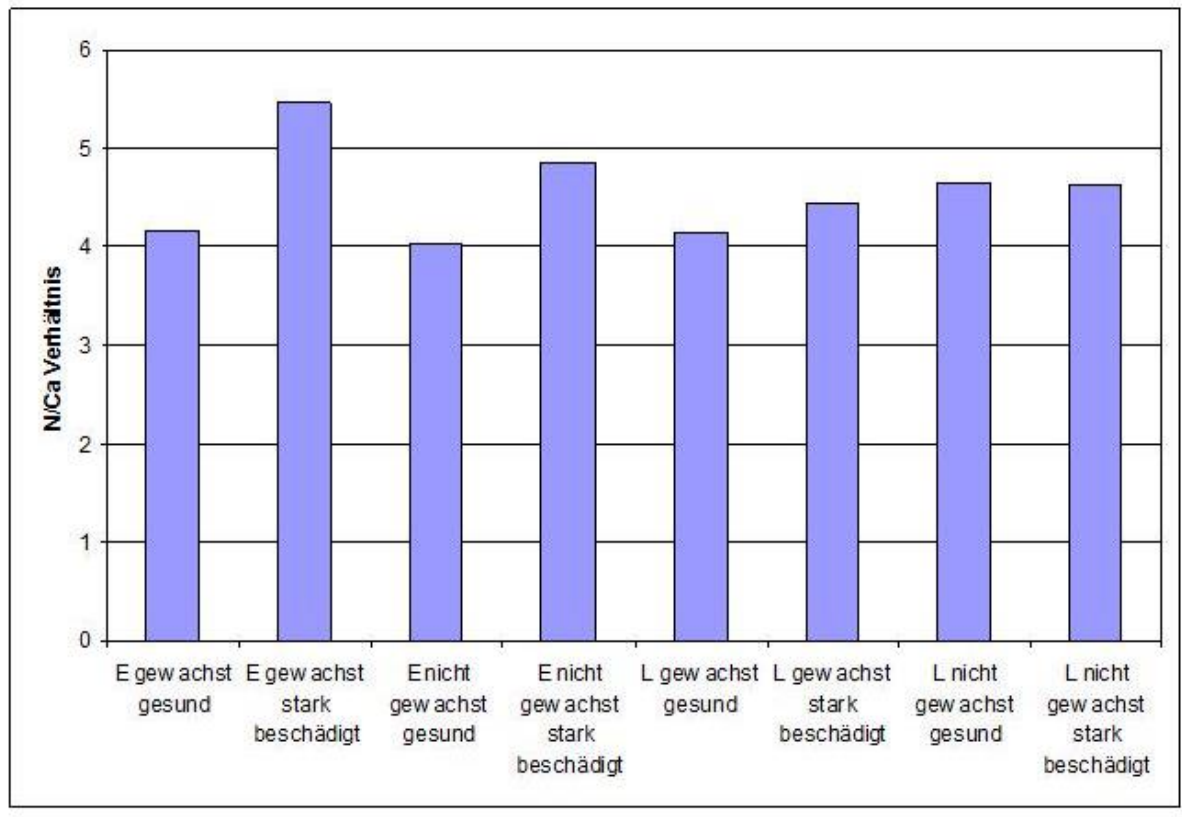

Abb. 14: N/Ca Verhältnis in verschiedenen Herkünften: Vergleich von gesunden und mit Lentizellen-Schwäche befallenen Früchten. // N/Ca ratio of different origins: comparison of healthy and due to 'Lenticel Breakdown' damaged fruits. 


\section{(ㄷ) $(1)$}

Dieses Werk ist lizenziert unter einer Creative Commons Namensnennung - Nicht kommerziell 4.0 International Lizenz. Quest'opera è distribuita con Licenza Creative Commons Attribuzione - Non commerciale 4.0 Internazionale. This work is licensed under a Creative Commons Attribution - NonCommercial 4.0 International License.

Für alle Abbildungen und Tabellen ohne Nennung des Urhebers gilt: @ Versuchszentrum Laimburg.

Per tutte le immagini e tabelle senza menzione dell'artefice vale: (c) Centro di Sperimentazione Laimburg.

For all figures and tables without mention of the originator applies: @ Laimburg Research Centre. 